Article

\title{
Three New Triterpene Esters from Pumpkin (Cucurbita maxima) Seeds
}

\author{
Takashi Kikuchi, Shinsuke Ueda, Jokaku Kanazawa, Hiroki Naoe, Takeshi Yamada and \\ Reiko Tanaka *
}

Osaka University of Pharmaceutical Sciences, 4-20-1 Nasahara, Takatsuki, Osaka, 569-1094, Japan

* Author to whom correspondence should be addressed; E-Mail: tanakar@gly.oups.ac.jp;

Tel./Fax: +81-72-690-1084.

Received: 7 February 2014; in revised form: 27 March 2014 / Accepted: 8 April 2014 /

Published: 16 April 2014

\begin{abstract}
Three new multiflorane-type triterpene esters, i.e. $7 \alpha$-hydroxymultiflor- 8 -ene3 $\alpha$,29-diol 3-acetate-29-benzoate (1), 7 $\alpha$-methoxymultiflor-8-ene-3 $\alpha, 29$-diol 3,29-dibenzoate (2), and 7 $\beta$-methoxymultiflor-8-ene-3 $\alpha, 29$-diol 3,29-dibenzoate (3), were isolated from seeds of Cucurbita maxima, along with the known compound, multiflora-7,9(11)-diene3 $\alpha, 29$-diol 3,29-dibenzoate (4). Compound 1 exhibited melanogenesis inhibitory activities comparable with those of arbutin. In cytotoxicity assays, compounds $\mathbf{1}$ and $\mathbf{3}$ exhibited weak cytotoxicity, with $\mathrm{IC}_{50}$ values of $34.5-93.7 \mu \mathrm{M}$ against HL-60 and P388 cells.
\end{abstract}

Keywords: Cucurbita maxima; multiflorane-type triterpene; melanogenesis inhibitory activity; cytotoxic activity

\section{Introduction}

Pumpkins, including Cucurbita moschata, C. pepo, and C. maxima, are gourd squashes of the genus Cucurbita and the family Cucurbitaceae. Cucurbita moschata seeds have been used as an anthelmintic [1], and Cucurbita pepo seeds, as an anthelmintic and a diuretic [2]. The isolation of 3-p-aminobenzoyl

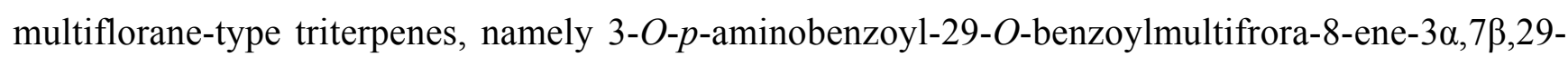
triol and 3-O-p-aminobenzoyl-29-O-benzoylmultifrora-7,9(11)-diene-3 $\alpha, 29$-diol, and 7-epi zucchini factor A, and debenzoyl zucchini factor B from C. pepo seeds has been reported [3,4].

Cucurbita maxima (English name: squash, pumpkin, Japanese name: Kabocha) is indigenous to the plateaus of central and south America, but is nowadays cultivated throughout the world. Its fruits, 
flowers, and seeds have been eaten as vegetables containing vitamins A, C, and E. Several triterpenes, such as cucurbita-5,24-dienol [5] and $\alpha$ - and $\beta$-amyrin [6], are present in the seeds of Cucurbita maxima. Additionally, it was demonstrated that the seeds and flowers of Cucurbita maxima contain sterols [6-8]. Recently we have reported the isolation of six multiflorane-type triterpenes including three new triterpenes: 7 $\alpha$-methoxymultiflor-8-ene-3 $\alpha, 29$-diol 3-acetate-29-benzoate, 7-oxomultiflor-8ene-3 $\alpha, 29$-diol 3-acetate-29-benzoate, and multiflora-7,9(11)-diene-3 $\alpha, 29$-diol 3-p-hydroxybenzoate29-benzoate, from seeds of C. maxima produced in Japan, and the melanogenesis inhibitory and cytotoxic activities of these compounds [9]. In a continuing study to explore new compounds possessing potent biological activities from $C$. maxima seeds, we have isolated four multiflorane-type triterpenes from seeds of $C$. maxima produced in India, and determined the structures of three new compounds: $7 \alpha$-hydroxymultiflor-8-ene-3 $\alpha, 29$-diol 3-acetate-29-benzoate (1) $7 \alpha$-methoxymultiflor-8ene-3 $\alpha, 29$-diol 3,29-dibenzoate (2), and 7 $\beta$-methoxymultiflor-8-ene-3 $\alpha, 29$-diol 3,29-dibenzoate (3). In addition, 1-3, were evaluated for inhibitory effects on $\alpha-\mathrm{MSH}$-induced melanogenesis in B16 melanomas, and cytotoxic activities against the HL-60 and P388 leukemia cell lines.

\section{Results and Discussion}

Four multiflorane-type triterpenes, including three new compounds, i.e. $7 \alpha$-hydroxymultiflor-8-ene-

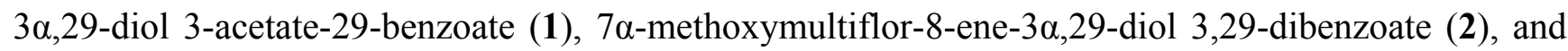
7 $\beta$-methoxymultiflor-8-ene-3 $\alpha, 29$-diol 3,29-dibenzoate (3), were isolated from the $\mathrm{MeOH}$ extract of $C$. maxima seeds (Figure 1). The known compound, i.e. multiflora-7,9(11)-diene-3 $\alpha, 29$-diol 3,29dibenzoate (4), was identified by comparing its MS and ${ }^{1} \mathrm{H}-\mathrm{NMR}$ data with published values [10].

Figure 1. Chemical structures of isolated compounds 1-4.

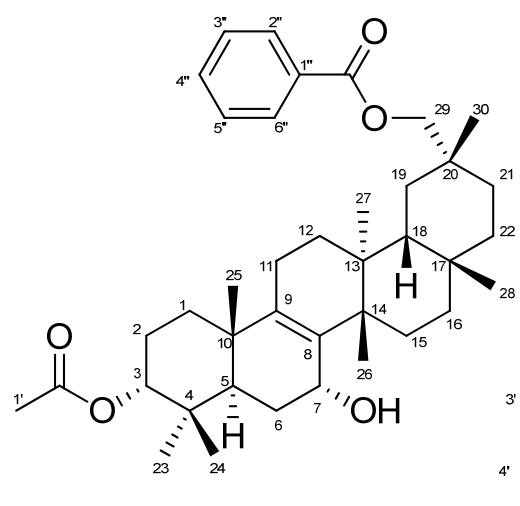

1

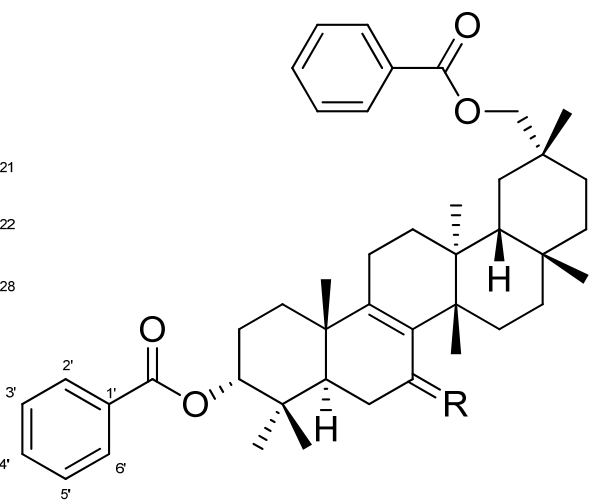

2: $\mathrm{R}=\alpha-\mathrm{OMe}, \beta-\mathrm{H}$ 3: $\mathrm{R}=\alpha-\mathrm{H}, \beta-\mathrm{OMe}$

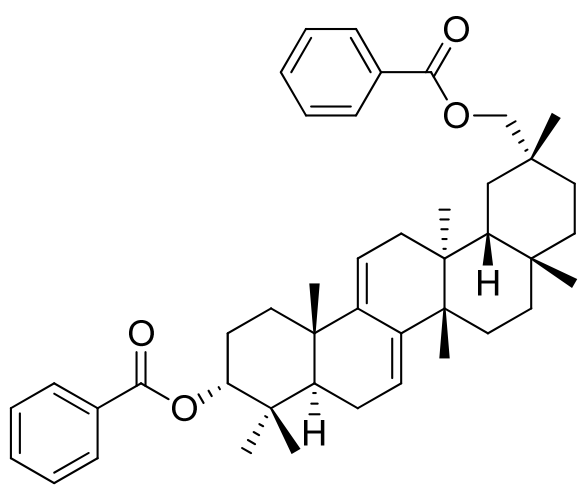

4

Compound 1 exhibited a $\left[\mathrm{M}-\mathrm{H}_{2} \mathrm{O}\right]^{+}$ion in the HREIMS data at $m / z 586.4019$ compatible with the molecular formula $\mathrm{C}_{39} \mathrm{H}_{54} \mathrm{O}_{4}$ (calcd. 586.4023), therefore it was suggested that the molecular formula of 1 is $\mathrm{C}_{39} \mathrm{H}_{56} \mathrm{O}_{5}$. The IR spectrum showed the presence of a hydroxy group $\left(v_{\max } 3437 \mathrm{~cm}^{-1}\right)$ and ester carbonyl groups $\left(v_{\max } 1718,1272,1244 \mathrm{~cm}^{-1}\right)$. The ${ }^{1} \mathrm{H}$ and ${ }^{13} \mathrm{C}-\mathrm{NMR}$ spectra (Table 1) displayed signals for seven tertiary methyl groups $\left[\delta_{\mathrm{H}} 0.89,0.91,0.92,1.03,1.06,1.10,1.12\right.$ (each s)], an oxymethylene $\left[\delta_{\mathrm{H}} 4.14\left(2 \mathrm{H}\right.\right.$, brs); $\left.\delta_{\mathrm{C}} 72.5(\mathrm{t})\right]$, two oxymethines [ $\delta_{\mathrm{H}} 4.16(\mathrm{brs}), 4.69(\mathrm{t}) ; \delta_{\mathrm{C}} 64.3(\mathrm{~d})$, $77.4(\mathrm{~d})]$, a tetrasubstituted olefin $\left[\delta_{\mathrm{C}} 136.4(\mathrm{~s}), 140.1(\mathrm{~s})\right]$, an acetoxy group $\left[\delta_{\mathrm{H}} 2.07(\mathrm{~s}) ; \delta_{\mathrm{C}} 21.4(\mathrm{q})\right.$, 
$170.9(\mathrm{~s})$ ], and a benzoyl group [ $\delta_{\mathrm{H}} 7.47(\mathrm{tt}), 7.58(\mathrm{tt}), 8.07(\mathrm{dd}) ; \delta_{\mathrm{C}} 128.4(\mathrm{~d}), 129.4(\mathrm{~d}), 130.6(\mathrm{~s})$, 132.9 (d), 166.7 (s)]. The ${ }^{1} \mathrm{H}$ - and ${ }^{13} \mathrm{C}$-NMR spectra are similar to those of 3-O-p-aminobenzoyl-29-Obenzoylmultiflor-8-ene-3 $\alpha, 7 \alpha, 29$-triol [3] except for the absence of a $p$-aminobenzoyl group at C-3 and existence of an acetyl group in 1. In the HMBC experiment, the following correlations were observed: Me-23 [ $\left.\delta_{\mathrm{H}} 0.89(\mathrm{~s})\right]$ to C-3 [ $\left.\delta_{\mathrm{c}} 77.4(\mathrm{~d})\right], \mathrm{C}-4, \mathrm{C}-5$, and C-24; Me-24 [ $\delta_{\mathrm{H}} 0.91$ (s)] to C-3, C-4, C-5, and Me-23; Me-25 [ $\left.\delta_{\mathrm{H}} 0.92(\mathrm{~s})\right]$ to C-1, C-5, C-9 [ $\left.\delta_{\mathrm{C}} 140.1(\mathrm{~s})\right]$, and C-10; Me-26 [ $\delta_{\mathrm{H}} 1.03$ (s)] to C-8 [ $\left.\delta_{\mathrm{C}} 136.4(\mathrm{~s})\right], \mathrm{C}-13, \mathrm{C}-14$, and C-15; Me-27 [ $\delta_{\mathrm{H}} 1.06$ (s)] to C-12, C-13, C-14, and C-18; Me-28 [ $\left.\delta_{\mathrm{H}} 1.12(\mathrm{~s})\right]$ to C-16, C-17, C-18, and C-22; $\mathrm{H}_{2}-29$ [ $\left.\delta_{\mathrm{H}} 4.14(2 \mathrm{H}, \mathrm{brs})\right]$ to C-19, C-20, C-21, C-30, and 29-Oㅡㅇ [ $\left.\delta_{\mathrm{C}} 166.7(\mathrm{~s})\right]$; Me-30 [ $\left.\delta_{\mathrm{H}} 1.10(\mathrm{~s})\right]$ to C-19, C-20, C-21, and C-29; H-3 [ $\left.\delta_{\mathrm{H}} 4.69(\mathrm{t})\right]$ to 3-Oㅡㅇ [ $\delta_{\mathrm{C}} 170.9(\mathrm{~s})$ ]; H-5 and H-6 $\alpha$ to $\mathrm{C}-7$ [ $\delta_{\mathrm{C}} 64.3(\mathrm{~d})$ ]; $\mathrm{H}-6 \alpha$ and $\mathrm{H}_{2}-11$ to $\mathrm{C}-8$ [ $\delta_{\mathrm{C}} 136.4(\mathrm{~s})$ ]; $\mathrm{H}-11$ and $\mathrm{H}-12 \delta$ to $\mathrm{C}-9$ [ $\delta_{\mathrm{C}} 140.1$ (s)]. In the ${ }^{1} \mathrm{H}-{ }^{1} \mathrm{H}$ COSY experiment, $\mathrm{H}-7$ [ $\delta_{\mathrm{H}} 4.16$ (brs)] correlated with $\mathrm{H}_{2}-6$ [ $\left.\delta_{\mathrm{H}} 1.60,1.74\right]$ (Figure 2). The following significant NOE interactions were observed in 1: $\mathrm{H}-5 / \mathrm{H}-1 \alpha$, Me-27; Me-23/H-6 $\alpha$; Me-27/H-15 $\alpha, \mathrm{H}-22 \alpha$, and $\mathrm{H}_{2}-29 ; \mathrm{H}-2 \beta$ /Me-24, and Me-25;

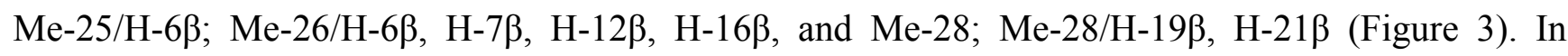
addition, the NOE correlations between $\mathrm{H}-7$ and Me-26 suggested that the hydroxy group at $\mathrm{C}-7$ is in the $\alpha$ (axial)-orientation (Figure 3). The configuration of the acetoxy group at C-3 was established as the $\alpha$ (axial)-orientation due to the coupling constants of $\mathrm{H}-3\left[\delta_{\mathrm{H}} 4.69\left(\mathrm{t}, J_{3 \beta .2 \alpha ; 3 \beta, 2 \beta}=3.0 \mathrm{~Hz}\right)\right]$ and NOEs between H-3 and Me-24. Therefore, the structure of 1 was determined to be as shown in Figure 1.

Figure 2. Key HMBC and ${ }^{1} \mathrm{H}-{ }^{1} \mathrm{H}$ COSY correlations of compound $\mathbf{1}$.

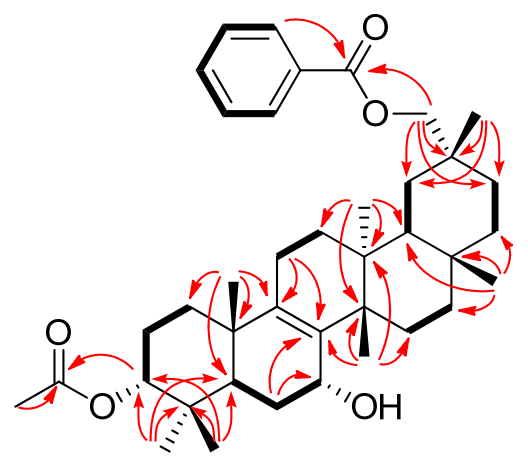

Figure 3. Selected NOE correlations of compound 1.

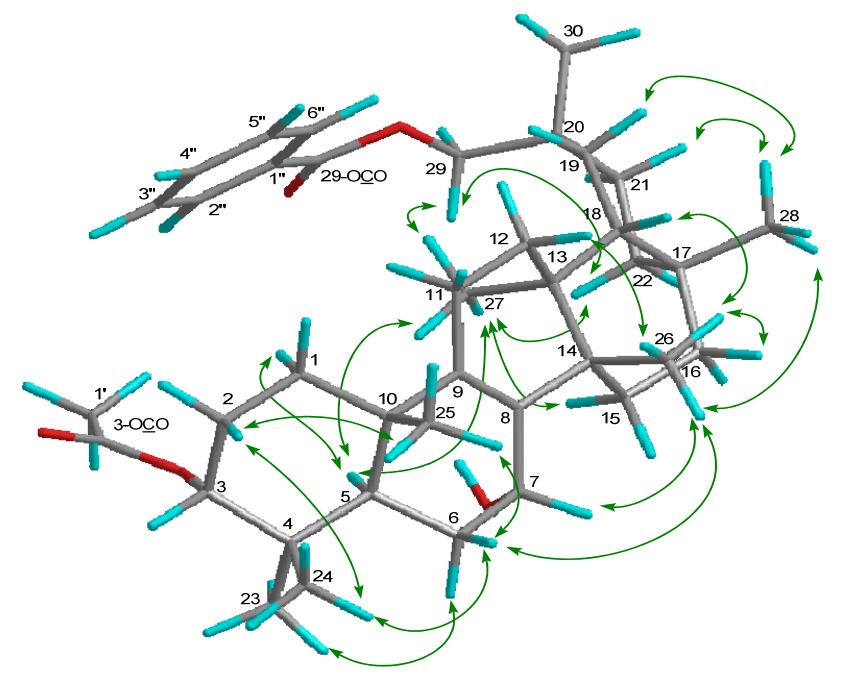


Table 1. ${ }^{1} \mathrm{H}(600 \mathrm{MHz})$ and ${ }^{13} \mathrm{C}(150 \mathrm{MHz})$ NMR spectroscopic data of compounds $\mathbf{1}-\mathbf{3}\left(\mathrm{CDCl}_{3}\right){ }^{\mathrm{a}}$.

\begin{tabular}{|c|c|c|c|c|c|c|c|c|c|c|c|c|c|c|c|}
\hline \multirow{3}{*}{$\begin{array}{c}\text { Position } \\
1\end{array}$} & \multicolumn{5}{|c|}{1} & \multicolumn{6}{|c|}{2} & \multicolumn{4}{|c|}{3} \\
\hline & \multicolumn{2}{|c|}{$\delta_{\mathrm{C}}$, type } & \multicolumn{3}{|c|}{$\delta_{\mathrm{H}}(J$ in $\mathrm{Hz})$} & \multicolumn{3}{|c|}{$\delta_{\mathrm{C}}$, type } & \multicolumn{2}{|c|}{$\delta_{\mathrm{H}}(J$ in $\mathrm{Hz})$} & \multicolumn{4}{|c|}{$\delta_{\mathrm{C}}$, type } & \multirow{2}{*}{$\frac{\delta_{\mathrm{H}}(\boldsymbol{J} \text { in } \mathbf{H z})}{\mathrm{m}}$} \\
\hline & 29.6 & $\mathrm{t}$ & $\alpha$ & 1.33 & $\mathrm{~m}$ & 29.8 & $\mathrm{t}$ & $\alpha$ & 1.48 & $\mathrm{~m}$ & 30.6 & $\mathrm{t}$ & $\alpha$ & 1.40 & \\
\hline & & & $\beta$ & 1.47 & $\mathrm{~m}$ & & & $\beta$ & 1.53 & $\mathrm{~m}$ & & & $\beta$ & 1.52 & $\mathrm{~m}$ \\
\hline \multirow[t]{2}{*}{2} & 23.3 & $\mathrm{t}$ & $\alpha$ & 1.66 & $\mathrm{~m}$ & 23.6 & $\mathrm{t}$ & $\alpha$ & 1.80 & $\mathrm{~m}$ & 23.3 & $\mathrm{t}$ & $\alpha$ & 1.80 & $\mathrm{~m}$ \\
\hline & & & $\beta$ & 1.88 & $\mathrm{~m}$ & & & $\beta$ & 1.98 & $\mathrm{~m}$ & & & $\beta$ & 1.97 & $\mathrm{~m}$ \\
\hline 3 & 77.4 & $\mathrm{~d}$ & & 4.69 & $t(3.0)$ & 78.1 & $\mathrm{~d}$ & & 4.95 & $\mathrm{t}(2.9)$ & 78.2 & $\mathrm{~d}$ & & 4.91 & $t(3.0)$ \\
\hline 4 & 36.3 & $\mathrm{~s}$ & & & & 36.7 & $\mathrm{~s}$ & & & & 37.0 & $\mathrm{~s}$ & & & \\
\hline 5 & 39.6 & $\mathrm{~d}$ & & 1.91 & $\mathrm{~m}$ & 39.9 & $\mathrm{~d}$ & & 2.19 & $\mathrm{dd}(12.6,1.2)$ & 44.1 & $\mathrm{~d}$ & & 1.70 & $\mathrm{~m}$ \\
\hline \multirow[t]{2}{*}{6} & 28.8 & $\mathrm{t}$ & $\alpha$ & 1.74 & $\mathrm{~m}$ & 22.5 & $\mathrm{t}$ & $\alpha$ & 1.95 & $\mathrm{~m}$ & 25.3 & $\mathrm{t}$ & $\alpha$ & 2.21 & $\mathrm{~m}$ \\
\hline & & & $\beta$ & 1.60 & $\mathrm{~m}$ & & & $\beta$ & 1.34 & $\mathrm{~m}$ & & & $\beta$ & 1.51 & $\mathrm{~m}$ \\
\hline 7 & 64.3 & $\mathrm{~d}$ & & 4.16 & brs & 73.8 & $\mathrm{~d}$ & & 3.54 & brs & 78.8 & $\mathrm{~d}$ & & 3.98 & $\operatorname{brt}(7.6)$ \\
\hline 8 & 136.4 & $\mathrm{~s}$ & & & & 135.3 & $\mathrm{~s}$ & & & & 136.7 & $\mathrm{~s}$ & & & \\
\hline 9 & 140.1 & $\mathrm{~s}$ & & & & 139.7 & $\mathrm{~s}$ & & & & 140.3 & $\mathrm{~s}$ & & & \\
\hline 10 & 38.6 & $\mathrm{~s}$ & & & & 38.6 & $\mathrm{~s}$ & & & & 38.2 & $\mathrm{~s}$ & & & \\
\hline \multirow[t]{2}{*}{11} & 20.9 & $\mathrm{t}$ & & 1.93 & $2 \mathrm{H}, \mathrm{m}$ & 20.9 & $\mathrm{t}$ & & 1.97 & $2 \mathrm{H}, \mathrm{m}$ & 20.8 & $\mathrm{t}$ & $\alpha$ & 2.03 & $\mathrm{~m}$ \\
\hline & & & & & & & & & & & & & $\beta$ & 1.91 & $\mathrm{~m}$ \\
\hline \multirow[t]{2}{*}{12} & 31.19 & $\mathrm{t}$ & $\alpha$ & 1.34 & $\mathrm{~m}$ & 31.3 & $\mathrm{t}$ & $\alpha$ & 1.35 & $\mathrm{~m}$ & 30.7 & $\mathrm{t}$ & $\alpha$ & 1.51 & $\mathrm{~m}$ \\
\hline & & & $\beta$ & 1.61 & $\mathrm{~m}$ & & & $\beta$ & 1.61 & $\mathrm{~m}$ & & & $\beta$ & 1.40 & $\mathrm{~m}$ \\
\hline 13 & 36.8 & $\mathrm{~s}$ & & & & 37.0 & $\mathrm{~s}$ & & & & 38.2 & $\mathrm{~s}$ & & & \\
\hline 14 & 41.9 & $\mathrm{~s}$ & & & & 41.8 & $\mathrm{~s}$ & & & & 40.9 & $\mathrm{~s}$ & & & \\
\hline \multirow[t]{2}{*}{15} & 26.1 & $\mathrm{t}$ & $\alpha$ & 2.05 & $\mathrm{~m}$ & 25.4 & $\mathrm{t}$ & $\alpha$ & 2.19 & $\mathrm{~m}$ & 26.3 & $\mathrm{t}$ & $\alpha$ & 1.78 & $\mathrm{~m}$ \\
\hline & & & $\beta$ & 1.50 & $\mathrm{~m}$ & & & $\beta$ & 1.26 & $\mathrm{~m}$ & & & $\beta$ & 1.83 & $\mathrm{~m}$ \\
\hline \multirow[t]{2}{*}{16} & 36.9 & $\mathrm{t}$ & $\alpha$ & 1.54 & $\mathrm{~m}$ & 36.9 & $\mathrm{t}$ & $\alpha$ & 1.56 & $\mathrm{~m}$ & 36.5 & $\mathrm{t}$ & $\alpha$ & 1.61 & $\mathrm{~m}$ \\
\hline & & & $\beta$ & 1.67 & $\mathrm{~m}$ & & & $\beta$ & 1.61 & $\mathrm{~m}$ & & & $\beta$ & 1.53 & $\mathrm{~m}$ \\
\hline 17 & 31.2 & $\mathrm{~s}$ & & & & 31.1 & $\mathrm{~s}$ & & & & 31.2 & $\mathrm{~s}$ & & & \\
\hline 18 & 44.4 & $\mathrm{~d}$ & & 1.61 & $\mathrm{~m}$ & 44.0 & $\mathrm{~d}$ & & 1.60 & $\mathrm{~m}$ & 42.8 & $\mathrm{~d}$ & & 1.66 & $\mathrm{~m}$ \\
\hline \multirow[t]{2}{*}{19} & 28.4 & $\mathrm{t}$ & $\alpha$ & 1.90 & $\mathrm{~m}$ & 28.8 & $\mathrm{t}$ & $\alpha$ & 1.86 & $\mathrm{~m}$ & 29.8 & $\mathrm{t}$ & $\alpha$ & 1.40 & $\mathrm{~m}$ \\
\hline & & & $\beta$ & 1.56 & $\mathrm{~m}$ & & & $\beta$ & 1.49 & $\mathrm{~m}$ & & & $\beta$ & 1.50 & $\mathrm{~m}$ \\
\hline 20 & 31.7 & $\mathrm{~s}$ & & & & 31.9 & $\mathrm{~s}$ & & & & 32.2 & $\mathrm{~s}$ & & & \\
\hline
\end{tabular}


Table 1. Cont.

\begin{tabular}{|c|c|c|c|c|c|c|c|c|c|c|c|c|c|c|c|}
\hline \multirow{3}{*}{$\frac{\text { Position }}{21}$} & \multicolumn{5}{|c|}{1} & \multicolumn{5}{|c|}{2} & \multicolumn{5}{|c|}{3} \\
\hline & \multicolumn{2}{|c|}{$\delta_{C}$, type } & & \multicolumn{2}{|c|}{$\delta_{H}(J$ in $\mathbf{H z})$} & \multicolumn{2}{|c|}{$\delta_{C}$, type } & \multicolumn{3}{|c|}{$\delta_{H}(J$ in $\mathbf{H z})$} & \multicolumn{2}{|c|}{$\delta_{C}$, type } & & \multicolumn{2}{|c|}{$\delta_{\mathrm{H}}(J$ in $\mathrm{Hz})$} \\
\hline & 30.1 & $\mathrm{t}$ & $\alpha$ & 1.47 & $\mathrm{~m}$ & 29.9 & $\mathrm{t}$ & $\alpha$ & 1.48 & $\mathrm{~m}$ & 29.1 & $\mathrm{t}$ & & 1.52 & $2 \mathrm{H}, \mathrm{m}$ \\
\hline & & & $\beta$ & 1.59 & $\mathrm{~m}$ & & & $\beta$ & 1.53 & $\mathrm{~m}$ & & & & & \\
\hline \multirow[t]{2}{*}{22} & 35.0 & $\mathrm{t}$ & $\alpha$ & 1.87 & $\mathrm{~m}$ & 35.6 & $\mathrm{t}$ & $\alpha$ & 1.84 & $\mathrm{~d}(4.4)$ & 37.1 & $\mathrm{t}$ & $\alpha$ & 1.68 & $\mathrm{~m}$ \\
\hline & & & $\beta$ & 0.97 & $\mathrm{~m}$ & & & $\beta$ & 0.96 & $\mathrm{~m}$ & & & $\beta$ & 1.01 & $\mathrm{~m}$ \\
\hline 23 & 27.4 & $\mathrm{q}$ & & 0.89 & $\mathrm{~s}$ & 27.5 & $\mathrm{q}$ & & 0.97 & $\mathrm{~s}$ & 27.6 & $\mathrm{q}$ & & 0.96 & $\mathrm{~s}$ \\
\hline 24 & 22.1 & $\mathrm{q}$ & & 0.91 & $\mathrm{~s}$ & 22.4 & $\mathrm{q}$ & & 1.00 & $\mathrm{~s}$ & 21.7 & $\mathrm{q}$ & & 1.02 & $\mathrm{~s}$ \\
\hline 25 & 18.1 & $\mathrm{q}$ & & 0.92 & $\mathrm{~s}$ & 18.2 & $\mathrm{q}$ & & 0.98 & $\mathrm{~s}$ & 20.2 & $\mathrm{q}$ & & 1.10 & $\mathrm{~s}$ \\
\hline 26 & 25.1 & $\mathrm{q}$ & & 1.03 & $\mathrm{~s}$ & 26.0 & $\mathrm{q}$ & & 1.05 & $\mathrm{~s}$ & 27.8 & $\mathrm{q}$ & & 1.29 & $\mathrm{~s}$ \\
\hline 27 & 19.0 & $\mathrm{q}$ & & 1.06 & $\mathrm{~s}$ & 19.0 & $\mathrm{q}$ & & 1.082 & $\mathrm{~s}$ & 18.0 & $\mathrm{q}$ & & 0.95 & $\mathrm{~s}$ \\
\hline 28 & 31.17 & $\mathrm{q}$ & & 1.12 & $\mathrm{~s}$ & 31.3 & $\mathrm{q}$ & & 1.13 & $\mathrm{~s}$ & 30.7 & $\mathrm{q}$ & & 1.17 & $\mathrm{~s}$ \\
\hline \multirow[t]{2}{*}{29} & 72.5 & $\mathrm{t}$ & & 4.14 & $2 \mathrm{H}, \mathrm{brs}$ & 72.9 & $\mathrm{t}$ & A & 4.08 & $\mathrm{~d}(10.8)$ & 74.0 & $\mathrm{t}$ & A & 4.05 & $\mathrm{~d}(10.6)$ \\
\hline & & & & & & & & $\mathrm{B}$ & 4.16 & $\mathrm{~d}(10.8)$ & & & B & 4.11 & $\mathrm{~d}(10.6)$ \\
\hline 30 & 30.6 & $\mathrm{q}$ & & 1.10 & $\mathrm{~s}$ & 29.8 & $\mathrm{q}$ & & 1.084 & $\mathrm{~S}$ & 28.1 & $\mathrm{q}$ & & 1.12 & $\mathrm{~S}$ \\
\hline $3-\mathrm{OCO}$ & 170.9 & $\mathrm{~s}$ & & & & 166.3 & $\mathrm{~s}$ & & & & 165.9 & $\mathrm{~s}$ & & & \\
\hline $1^{\prime}$ & 21.4 & $q$ & & 2.07 & $\mathrm{~s}$ & $130.8^{\mathrm{b}}$ & $\mathrm{s}$ & & & & 130.6 & $\mathrm{~s}$ & & & \\
\hline $2^{\prime}, 6^{\prime}$ & & & & & & $129.6^{\mathrm{c}}$ & $\mathrm{d}$ & & $8.05^{b}$ & $\mathrm{dd}(7.4,1.4)$ & 129.4 & $\mathrm{~d}$ & & 7.99 & $\mathrm{dd}(7.4,1.4)$ \\
\hline $3^{\prime}, 5^{\prime}$ & & & & & & $128.4^{\mathrm{d}}$ & $\mathrm{d}$ & & $7.45^{\mathrm{c}}$ & $\mathrm{tt}(7.4,1.4)$ & 128.5 & $\mathrm{~d}$ & & 7.45 & $\operatorname{tt}(7.4,1.4)$ \\
\hline $4^{\prime}$ & & & & & & $132.7^{\mathrm{e}}$ & $\mathrm{d}$ & & $7.55^{\mathrm{d}}$ & $\mathrm{tt}(7.4,1.4)$ & 132.8 & $\mathrm{~d}$ & & 7.56 & $\mathrm{tt}(7.4,1.4)$ \\
\hline 29-O드 & 166.7 & $\mathrm{~s}$ & & & & 166.6 & $\mathrm{~s}$ & & & & 166.8 & $\mathrm{~s}$ & & & \\
\hline 1" & 130.6 & $\mathrm{~s}$ & & & & $130.7^{\mathrm{b}}$ & $\mathrm{s}$ & & & & 130.9 & $\mathrm{~s}$ & & & \\
\hline 2", 6" & 129.4 & $\mathrm{~d}$ & & 8.07 & $\mathrm{dd}(7.4,1.2)$ & $129.4^{\mathrm{c}}$ & $\mathrm{d}$ & & $8.04^{b}$ & $\mathrm{dd}(7.4,1.4)$ & 129.5 & $\mathrm{~d}$ & & 8.04 & $\mathrm{dd}(7.3,1.7)$ \\
\hline 3", 5" & 128.4 & $\mathrm{~d}$ & & 7.47 & $\mathrm{tt}(7.4,1.2)$ & $128.3^{\mathrm{d}}$ & $\mathrm{d}$ & & $7.43^{c}$ & $\mathrm{tt}(7.4,1.4)$ & 128.4 & $\mathrm{~d}$ & & 7.43 & $\mathrm{tt}(7.3,1.7)$ \\
\hline 4" & 132.9 & $\mathrm{~d}$ & & 7.58 & $\mathrm{tt}(7.4,1.2)$ & $132.6^{\mathrm{e}}$ & $\mathrm{d}$ & & $7.54^{\mathrm{d}}$ & $\mathrm{tt}(7.4,1.4)$ & 132.7 & d & & 7.55 & $\mathrm{tt}(7.3,1.7)$ \\
\hline 7-OMe & & & & & & 54.9 & $\mathrm{q}$ & & 3.24 & $\mathrm{~s}$ & 55.0 & $\mathrm{q}$ & & 3.35 & $\mathrm{~s}$ \\
\hline
\end{tabular}

${ }^{a}$ Assignments were based on ${ }^{1} \mathrm{H}^{-1} \mathrm{H}$ COSY, HMQC, HMBC and NOESY supectroscopic data. ${ }^{\text {b-e }}$ Interchengeable. 
Compound 2 exhibited a $[\mathrm{M}]^{+}$ion in the HREIMS data at $m / z 680.4447$ compatible with the molecular formula $\mathrm{C}_{45} \mathrm{H}_{60} \mathrm{O}_{5}$ (calcd. 680.4441). The IR spectrum showed absorption indicating ester carbonyl groups $\left(v_{\max } 1717,1274 \mathrm{~cm}^{-1}\right)$. The ${ }^{1} \mathrm{H}$ - and ${ }^{13} \mathrm{C}-\mathrm{NMR}$ spectra (Table 1) displayed signals for seven tertiary methyl groups $\left[\delta_{\mathrm{H}} 0.97,0.98,1.00,1.05,1.082,1.084,1.13\right.$ (each s)], an oxymethylene [ $\delta_{\mathrm{H}} 4.08,4.16\left(\right.$ each d); $\left.\delta_{\mathrm{C}} 72.9(\mathrm{t})\right]$, two oxymethines [ $\left.\delta_{\mathrm{H}} 3.54(\mathrm{brs}), 4.95(\mathrm{t}) ; \delta_{\mathrm{C}} 73.8(\mathrm{~d}), 78.1(\mathrm{~d})\right]$, a tetrasubstituted olefin $\left[\delta_{\mathrm{C}} 135.3(\mathrm{~s}), 139.7(\mathrm{~s})\right]$, and two benzoyl groups [ $\delta_{\mathrm{H}} 7.43(\mathrm{tt}), 7.45(\mathrm{tt}), 7.54(\mathrm{tt})$, $7.55(\mathrm{tt}), 8.04(\mathrm{dd}), 8.05(\mathrm{dd}) ; \delta_{\mathrm{C}} 128.3(\mathrm{~d}), 128.4(\mathrm{~d}), 129.4(\mathrm{~d}), 129.6(\mathrm{~d}), 130.7(\mathrm{~s}), 130.8(\mathrm{~s}), 132.6$ (d), 132.7 (d), 166.3 (s), 166.6 (s)]. The above data suggested that the structure of 2 is similar to that of $7 \alpha$-methoxymultiflor-8-ene-3 $\alpha, 29$-diol 3-acetate-29-benzoate [9], except for the lack of the 3-O-acetyl group and the existence of a 3-O-benzoyl group. In the HMBC experiment, the following correlations were observed: $\mathrm{H}_{2}-29$ [ $\delta_{\mathrm{H}} 4.08,4.16$ (each d)] to 29-Oㅡㅇ [ $\left.\delta_{\mathrm{C}} 166.6(\mathrm{~s})\right] ; \mathrm{H}-3$ [ $\left.\delta_{\mathrm{H}} 4.95(\mathrm{t})\right]$ to 3-OCO

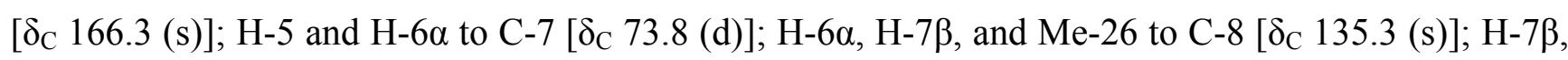
$\mathrm{H}_{2}-11$ and Me-25 to C-9 [ $\delta_{\mathrm{C}} 139.7$ (s)]. In the ${ }^{1} \mathrm{H}-{ }^{1} \mathrm{H}$ COSY experiment, $\mathrm{H}-7$ [ $\delta_{\mathrm{H}} 3.54$ (brs)] correlated with $\mathrm{H}_{2}-6\left[\delta_{\mathrm{H}} 1.34,1.95\right]$ (Figure 4). Additionally, the NOEs were observed H-7/H-15ß, Me-26; 7-OMe/H-5 and $\mathrm{H}-15 \alpha$; suggested that the methoxy group at $\mathrm{C}-7$ was in the $\alpha$-orientation (Figure 5). The configuration of the acetoxy group at $\mathrm{C}-3$ was established as the $\alpha$-orientation due to the significant NOEs between H-3 and Me-24, and the coupling constants of H-3 [ $\left.\delta_{\mathrm{H}} 4.95(\mathrm{t}, J=2.9 \mathrm{~Hz})\right]$. Therefore, $\mathbf{2}$ was established as shown in Figure 1.

Figure 4. Key HMBC and ${ }^{1} \mathrm{H}-{ }^{1} \mathrm{H}$ COSY correlations of compound 2.

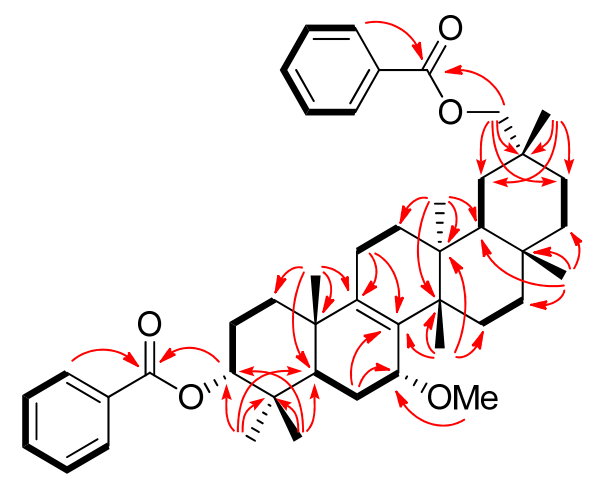

Figure 5. Selected NOE correlations of compound 2.

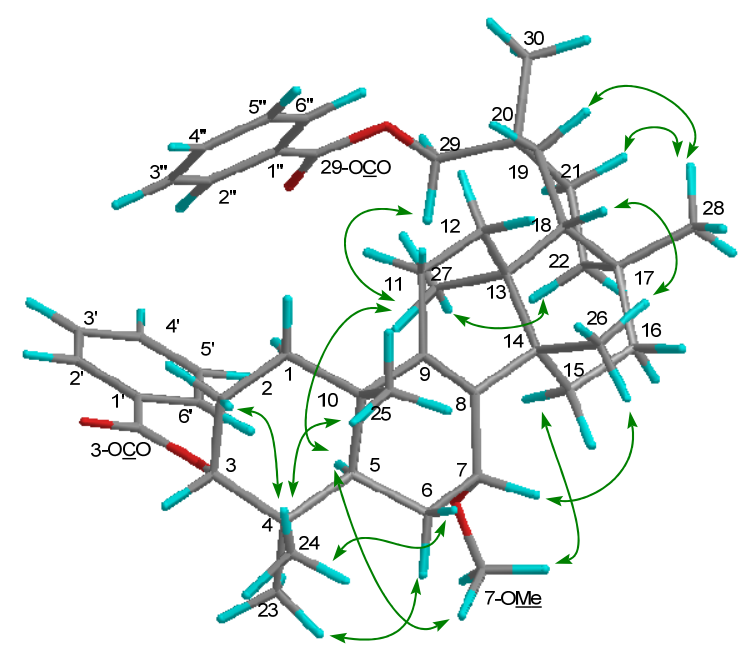


Compound 3 exhibited a $[\mathrm{M}]^{+}$ion in the HREIMS data at $\mathrm{m} / \mathrm{z} 680.4446$ compatible with the molecular formula $\mathrm{C}_{45} \mathrm{H}_{60} \mathrm{O}_{5}$ (calcd. 680.4440). The IR spectrum showed absorption indicating ester carbonyl groups $\left(v_{\max } 1717,1272 \mathrm{~cm}^{-1}\right)$. The ${ }^{1} \mathrm{H}$ - and ${ }^{13} \mathrm{C}$-NMR spectra (Table 1$)$ displayed signals for seven tertiary methyl groups $\left[\delta_{\mathrm{H}} 0.95,0.96,1.02,1.10,1.12,1.17,1.29\right.$ (each s)], an oxymethylene $\left[\delta_{\mathrm{H}}\right.$ 4.05, $4.11\left(\right.$ each d); $\delta_{\mathrm{C}} 74.0(\mathrm{t})$ ], two oxymethines [ $\delta_{\mathrm{H}} 3.98(\mathrm{brt}), 4.91(\mathrm{t}) ; \delta_{\mathrm{C}} 78.2(\mathrm{~d}), 78.8(\mathrm{~d})$ ], a tetrasubstituted olefin $\left[\delta_{\mathrm{C}} 136.7(\mathrm{~s}), 140.3(\mathrm{~s})\right]$, and two benzoyl group [ $\delta_{\mathrm{H}} 7.43(\mathrm{tt}), 7.45(\mathrm{tt}), 7.55(\mathrm{tt})$, $7.56(\mathrm{tt}), 7.99(\mathrm{dd}), 8.04(\mathrm{dd}) ; \delta_{\mathrm{C}} 128.4(\mathrm{~d}), 128.5(\mathrm{~d}), 129.4$ (d), 129.5 (d), 130.6 (s), 130.9 (s), 132.7 (d), 132.8 (d), 165.9 (s), 166.8 (s)]. The ${ }^{1} \mathrm{H}$ - and ${ }^{13} \mathrm{C}-\mathrm{NMR}$ spectra of $\mathbf{3}$ were very similar to those of $\mathbf{2}$, except for the H-7 signal [ $\delta_{\mathrm{H}} 3.98$ (brt, $J=7.6 \mathrm{~Hz}$ ): $\delta_{\mathrm{C}} 78.8$ (d) in $\mathbf{3} ; \delta_{\mathrm{H}} 3.54$ (brs): $\delta_{\mathrm{C}} 73.8$ (d) in 2 ]. The coupling constants of H-7, and the NOE correlations of H-7/H-5 $\alpha$, and H-15 $\alpha$; 7-OMe/Me-26 suggested that the methoxy group at $\mathrm{C}-7$ is in the $\beta$ (equatorial)-orientation (Figure 6). The configuration of the benzoyl group at C-3 was established as the $\alpha$-orientation due to the coupling constants of H-3 $\left[\delta_{\mathrm{H}} 4.91(\mathrm{t}, J=3.0 \mathrm{~Hz})\right]$. The above data established that 3 was a $7 \beta$-methoxy epimer of 2 (Figure 1).

Figure 6. Selected NOE correlations of compound 3.

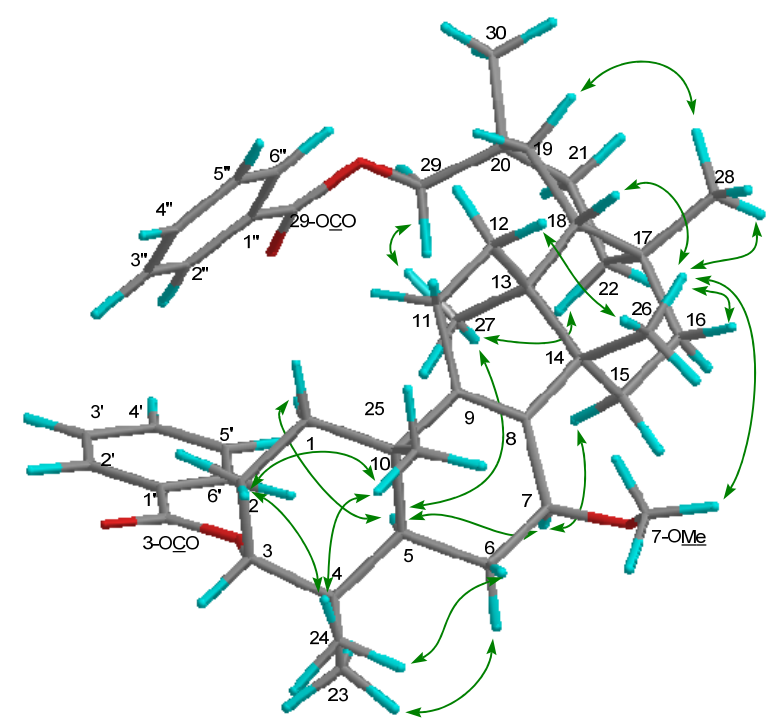

Multiflorane-type triterpenes are unusual, and most of them have been isolated from cucurbitaceae plants, such as Cucumis melo [11], Cucurbita pepo [3,4], Momordica cochinchinensis [12], and Trichosanthes kirilowii [13]. Only a few of their biological activities, such as anti-tumor promoting activities [14], anti-oxidant effects [15], cytotoxic activities [9,16], and melanogenesis inhibitory activities $[9,16]$, have been reported. In this study, we evaluated them for melanogenesis inhibitory effects and cytotoxic activities against cancer cell lines. Melanogenesis plays an important role to protect the skin from UV irradiation. However, overproduction of melanin causes esthetic and dermatological problems [17], thus, several hypopigmenting products have been developed [17]. In this study, three new multiflorane triterpenes 1-3 from C. maxima were evaluated for inhibitory activities against $\alpha$-MSH-induced melanogenesis in B16 melanomas (Table 2). To determine the safe concentration, cytotoxicities of compounds against B16 4A5 cells were examined by an MTT assay. Compound 2 did not exhibit cytotoxicity at 10-100 $\mu \mathrm{M}$. Compounds $\mathbf{1}$ and $\mathbf{3}$ showed no toxicities at 
$10 \mu \mathrm{M}$, although they decreased cell viabilities at higher concentrations (1: $88.0 \%$ at $30 \mu \mathrm{M}, 58.4 \%$ at $100 \mu \mathrm{M} ; 3: 86.3 \%$ at $30 \mu \mathrm{M}, 67.2 \%$ at $100 \mu \mathrm{M})$. In the melanogenesis inhibitory assay, compound 1 reduced melanin content $(88.5 \%)$ at a non-toxic concentration, $10 \mu \mathrm{M}$. The melanogenesis inhibitory activity of compound 1 was comparable with that of the positive control, arbutin (melanin content $88.9 \%$ at $10 \mu \mathrm{M}$ ), which has been recognized as a useful depigmentation compound for skin whitening in the cosmetic industry [18]. These results suggested that compound $\mathbf{2}$ may be valuable as a potential skin-whitening agent. Compounds $2(10-100 \mu \mathrm{M})$ and $3(10 \mu \mathrm{M})$ did not show any melanogenesis inhibitory activities.

Table 2. Melanogenesis inhibitory activity and cytotoxicity in B16 mouse melanoma cells of multiflorane-type triterpenes isolated from Cucurbita maxima seeds a .

\begin{tabular}{|c|c|c|c|c|c|c|}
\hline \multirow{2}{*}{\multicolumn{2}{|c|}{ Compound }} & \multicolumn{4}{|c|}{ Conc. $(\mu \mathrm{M})$} & \multirow{2}{*}{$\mathrm{IC}_{50}(\boldsymbol{\mu M})$} \\
\hline & & 10 & 30 & 100 & 300 & \\
\hline \multirow{2}{*}{1} & melanin content & $88.5 \pm 2.7 * *$ & $65.8 \pm 1.7 * *$ & $35.7 \pm 0.8 * *$ & & 46.5 \\
\hline & cell viability & $105.4 \pm 4.5$ & $88.0 \pm 1.0$ & $58.4 \pm 7.7 * *$ & & $>100$ \\
\hline \multirow{2}{*}{2} & melanin content & $96.3 \pm 2.2$ & $105.5 \pm 4.5$ & $108.3 \pm 1.0$ & & $>100$ \\
\hline & cell viability & $103.4 \pm 3.6$ & $105.8 \pm 5.0$ & $104.9 \pm 7.7$ & & $>100$ \\
\hline \multirow{2}{*}{3} & melanin content & $96.9 \pm 9.2$ & $79.4 \pm 4.5 * *$ & $60.1 \pm 1.8 * *$ & & $>100$ \\
\hline & cell viability & $94.6 \pm 0.4$ & $86.3 \pm 2.4 *$ & $67.2 \pm 4.8^{* *}$ & & $>100$ \\
\hline \multirow{2}{*}{$4^{b}$} & melanin content & $98.4 \pm 3.2$ & $102.2 \pm 11.7$ & $95.4 \pm 8.4$ & & $>100$ \\
\hline & cell viability & $110.8 \pm 4.3$ & $103.0 \pm 8.2$ & $101.1 \pm 5.9$ & & $>100$ \\
\hline \multirow{2}{*}{ Arbutin $^{\mathrm{c}}$} & melanin content & $88.9 \pm 2.3 * *$ & $72.3 \pm 3.1 * *$ & $55.3 \pm 1.0 * *$ & $33.8 \pm 2.8 * *$ & 124.6 \\
\hline & cell viability & $100.0 \pm 2.7$ & $94.4 \pm 1.2$ & $89.9 \pm 0.3 * *$ & $81.9 \pm 3.2 * *$ & $>300$ \\
\hline
\end{tabular}

${ }^{a}$ Melanin content (\%) and cell viability (\%) were determined based on the absorbance at $450 \mathrm{~nm}$, and $540 \mathrm{~nm}$, respectively, by comparison with values for DMSO (100\%). Each value represents the mean \pm standard deviation (S.D.) of three determinations. Asterisks denote significant differences from control group, ${ }^{*} p<0.05,{ }^{*} p<<0.01$. The concentration of DMSO in the sample solution was $2 \mu \mathrm{L} / \mathrm{mL}$. ${ }^{\mathrm{b}}$ Melanogenesis inhibitory and cytotoxicity data from [9]. ${ }^{\mathrm{c}}$ Reference compound.

Three triterpenes and a reference compound, 5-fluorouracil (5-FU), were also evaluated for cytotoxic activities against human leukemia (HL-60) and murine leukemia (P388) cell lines by means of the MTT assay. Compounds $\mathbf{1}$ and $\mathbf{3}$ exhibited weak cytotoxicities against HL-60 (IC $\mathrm{I}_{50}$ 1:89.2 $\mu \mathrm{M}$; 3:64.6 $\mu \mathrm{M})$ and $\mathrm{P} 388\left(\mathrm{IC}_{50}\right.$ 1:93.7 $\left.\mu \mathrm{M} ; \mathbf{3 : 3 4 . 5} \mu \mathrm{M}\right)$. Compound 2 did not show activities against either cell line $\left(\mathrm{IC}_{50}\right.$ each $\left.>100 \mu \mathrm{M}\right)$. In our previous study, several mutiflorane-type triterpenes were evaluated for their cytotoxic activities, and they showed no or weak activities, except 7-oxomultiflor-8ene-3 $\alpha, 29$-diol 3-acetate-29-benzoate, having a conjugated enone [9]. Results of this and previous studies suggest that a conjugated enone moiety strengthens the cytotoxic activities of multifloranetype triterpenes.

\section{Experimental}

\subsection{General Experimental Procedures}

Chemicals and reagents were purchased as follows: fetal bovine serum (FBS) from Invitrogen (Carlsbad, CA, USA), 3-(4,5-dimethyl-2-thiazolyl)-2,5-diphenyl-2H-tetrazolium bromide (MTT) from 
Sigma-Aldrich Japan Co. (Tokyo, Japan), Roswell Park Memorial Institute (RPMI) 1640 medium, Dulbecco's modified Eagle's medium (D-MEM), and antibiotics from Nacalai tesque, Inc. (Kyoto, Japan). All other chemicals and reagents were of analytical grade. Melting points were determined on a Yanagimoto micro-melting point apparatus and are uncorrected. Optical rotations were measured with a JASCO DIP-1000 digital polarimeter. IR spectra were recorded on a Perkin-Elmer 1720X FTIR spectrophotometer. The ${ }^{1} \mathrm{H}-(600 \mathrm{MHz})$ and ${ }^{13} \mathrm{C}-(150 \mathrm{MHz}) \mathrm{NMR}$ spectra were recorded on an Agilent vnmrs600 instrument in $\mathrm{CDCl}_{3}$ with tetramethylsilane as the internal standard. The EIMS was recorded on a Hitachi $4000 \mathrm{H}$ double-focusing mass spectrometer $(70 \mathrm{eV})$. Silica gel (70-230 mesh, Merck, Darmstadt, Germany) and silica gel 60 (230-400 mesh, Nacalai tesque, Inc.) were used for column chromatography and medium-pressure liquid chromatography, respectively. HPLC was carried out on an $\mathrm{SiO}_{2}$ column [Cosmosil 5SL-II column (Nacalai tesque, Inc.), $25 \mathrm{~cm} \times 20 \mathrm{~mm}$ i.d.] at $25{ }^{\circ} \mathrm{C}$ with $n$-hexane/EtOAc [20:1 (HPLC system I), 10:1 (HPLC system II), and 5:1 (HPLC system III), flow rate $8.0 \mathrm{~mL} / \mathrm{min}$ ], and on ODS column [Cosmosil 5C 18 -MS-II column (Nacalai tesque, Inc.), $25 \mathrm{~cm} \times$ $20 \mathrm{~mm}$ i.d.] at $25{ }^{\circ} \mathrm{C}$ with $\mathrm{Me}_{2} \mathrm{CO}: \mathrm{H}_{2} \mathrm{O}$ [10:1 (HPLC system IV) and 9:1 (HPLC system V), flow rate $8.0 \mathrm{~mL} / \mathrm{min}]$.

\subsection{Plant Material}

The seeds of Cucurbita maxima, produced in India, were purchased from Takada Seeds Co., Ltd. (Osaka, Japan) in 2011. A voucher specimen was deposited in the Herbarium of the Laboratory of Medicinal Chemistry, Osaka University of Pharmaceutical Sciences.

\subsection{Extraction and Isolation}

The seeds of Cucurbita maxima $(10 \mathrm{~kg})$ produced in India, were subjected to extraction with $\mathrm{MeOH}$ under reflux (30 L, one week, four times). The $\mathrm{MeOH}$ extract $(310 \mathrm{~g})$ was then partitioned between $\mathrm{Et}_{2} \mathrm{O}$ and $\mathrm{H}_{2} \mathrm{O}$. The $\mathrm{Et}_{2} \mathrm{O}$-soluble fraction ( $150 \mathrm{~g}$ ) was subjected to $\mathrm{SiO}_{2}$ column chromatography (CC) $\left[\mathrm{SiO}_{2}(3.5 \mathrm{~kg}) ; \mathrm{CHCl}_{3} / \mathrm{MeOH}\right.$ 1:0, 10:1, 5:1, and 0:1 in increasing order of polarity] resulting in 9 fractions (Fr. A-I). Fr. B, eluted with $\mathrm{CHCl}_{3}$, was subjected to $\mathrm{SiO}_{2} \mathrm{CC}$ to yield 18 fractions, B1-B18. Preparative HPLC of B7 (42.5 mg) (HPLC system II), eluted with hexane/EtOAc (10:1), gave 4 (28.0 mg; $t_{\mathrm{R}} 23.2$ min). Fr. C, eluted with $\mathrm{CHCl}_{3}$, was subjected to $\mathrm{SiO}_{2} \mathrm{CC}$ to yield 24 fractions, C1-C24. Preparative HPLC of C5 (67.9 mg)(HPLC system II), eluted with hexane/EtOAc (10:1), gave Fr. C5-4 (44.4 mg; tR $11.2 \mathrm{~min})$, and then re-preparative HPLC gave $2\left(11.1 \mathrm{mg} ; \mathrm{t}_{\mathrm{R}} 36.0 \mathrm{~min}\right)(\mathrm{HPLC}$ system I). Preparative HPLC (HPLC system II) of C6 (64.0 mg), eluted with hexane/EtOAc (10:1), gave 15 fractions; C6-1-C6-15, and preparative HPLC (HPLC system II) of C7 (15.4 mg), eluted with hexane/EtOAc (10:1), gave 15 fractions; C7-1-C7-15. Preparative HPLC of C6-6 (2.9 mg; tR $13.0 \mathrm{~min})$ and C7-6 (0.4 mg) combination gave $3\left(1.0 \mathrm{mg}\right.$; $\left.\mathrm{t}_{\mathrm{R}} 59.0 \mathrm{~min}\right)(\mathrm{HPLC}$ system IV). Fr. E, eluted with $\mathrm{CHCl}_{3}$, was fractionated with $\mathrm{SiO}_{2} \mathrm{CC}$ to E1-E11. Preparative HPLC (HPLC system III) of E8 (119.8 mg), eluted with hexane/EtOAc (5:1), gave Fr. E8-10 (8.8 mg; $\left.\mathrm{t}_{\mathrm{R}} 34.8 \mathrm{~min}\right)$, and then re-preparative HPLC (HPLC system V) gave 1 (1.6 mg; $t_{R} 13.6 \mathrm{~min}$ ). 


\subsection{Product Characterization Data}

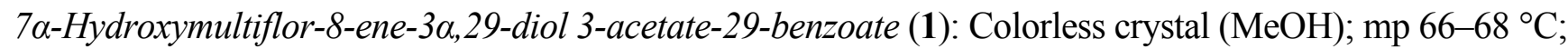
$[\alpha]_{D}^{22}-85.8\left(c=0.2, \mathrm{CHCl}_{3}\right)$; UV (EtOH) $\lambda_{\max }(\log \varepsilon) 205.0$ (3.80), 220.0 (3.94), 232.5 (3.98), 270.5 (3.41), 280.0 (3.32), 321.0 (2.87) nm; IR (KBr) $v_{\max }$ 3437, 2938, 2876, 1718, 1272, 1244, 1110, 750, 729, $710 \mathrm{~cm}^{-1} ;{ }^{1} \mathrm{H}$ and ${ }^{13} \mathrm{C}-\mathrm{NMR}$ data see Table 1; EIMS m/z $586\left[\mathrm{M}-\mathrm{H}_{2} \mathrm{O}\right]^{+}$(26), 540 (33), 527 (40), 511 (100), 389 (12), 387 (9), 253 (15), 225 (16); HREIMS m/z 586.4019 (calcd for $\mathrm{C}_{39} \mathrm{H}_{54} \mathrm{O}_{4}$ : 586.4023).

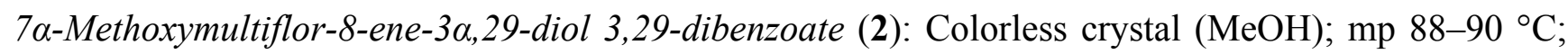
$[\alpha]_{D}^{28}-20.7\left(c=0.7, \mathrm{CHCl}_{3}\right)$; UV (EtOH) $\lambda_{\max }(\log \varepsilon) 238.5$ (3.70), 271.5 (3.21), 278.5 (3.11) nm; IR $(\mathrm{KBr}) v_{\max }: 2948,2883,1717,1456,1367,1314,1274,1113,1069,716 \mathrm{~cm}^{-1} ;{ }^{1} \mathrm{H}$ and ${ }^{13} \mathrm{C}-\mathrm{NMR}$ data see Table 1; EIMS m/z 680 (6) [M] $]^{+}, 648$ [M-MeOH] $^{+}$(12), 526 (25), 511 (100), 389 (8), 355 (10), 324 (8); HREIMS $m / z 680.4447$ (calcd for $\mathrm{C}_{45} \mathrm{H}_{60} \mathrm{O}_{5}$ : 680.4441).

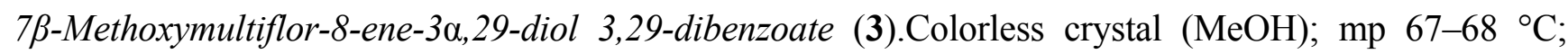
$[\alpha]_{D}^{22}-43.7\left(c=0.2, \mathrm{CHCl}_{3}\right)$; UV (EtOH) $\lambda_{\max }(\log \varepsilon) 206.5$ (3.86), 220.0 (3.97), 235.0 (4.00), 271.0 (3.29), 280.0 (3.16) nm; IR (KBr) $v_{\max }: 3437,1717,1272,1110,1028,975,711 \mathrm{~cm}^{-1} ;{ }^{1} \mathrm{H}$ and ${ }^{13} \mathrm{C}-\mathrm{NMR}$ data see Table 1; EIMS 680 (65) [M] $]^{+}, 665$ (38), $648[\mathrm{M}-\mathrm{MeOH}]^{+}$(14), 526 (29), 511 (100), 393 (18), 381 (18), 354 (28); HREIMS $m / z 680.4446[\mathrm{M}]^{+}$(calcd for $\mathrm{C}_{45} \mathrm{H}_{60} \mathrm{O}_{5}:$ : 680.4440).

\subsection{Cell Cultures}

The cell lines HL-60 (human leukemia) and P388 (murine leukemia) were grown in RPMI 1640 medium, while B16 4A5 cells were grown in D-MEM. The medium was supplemented with 10\% FBS and antibiotics (100 units/mL penicillin and $100 \mu \mathrm{g} / \mathrm{mL}$ streptomycin). The cells were incubated at $37^{\circ} \mathrm{C}$ in a $5 \% \mathrm{CO}_{2}$ humidified incubator.

\subsection{Determination of $B 164 A 5$ Cells Proliferation}

The assay of B16 4A5 cells proliferation was examined according to a method reported previously [9].

\subsection{Assay of Melanin Content}

The assay of melanin content was performed as described previously [9].

\subsection{Cytotoxicity Assay against Cancer Cell Lines}

The cytotoxicity assay was determined previously [19].

\section{Conclusions}

In this study, we isolated three new multiflorane-type triterpene esters, i.e. $7 \alpha$-hydroxymultiflor-8ene-3 $\alpha, 29$-diol 3-acetate-29-benzoate (1), 7 $\alpha$-methoxymultiflor-8-ene-3 $\alpha, 29$-diol 3,29-dibenzoate (2), and 7 $\beta$-methoxymultiflor-8-ene-3 $\alpha, 29$-diol 3,29-dibenzoate (3), from pumpkin seeds. Isolated compounds were evaluated for melanogenesis inhibitory and cytotoxic activities. In the melanogenesis 
inhibitory assay, we revealed that compound $\mathbf{1}$ possessed melanogenesis inhibitory activities comparable with arbutin at a non-toxic concentration. In a cytotoxicity assay against cancer cell lines, none of the compounds showed remarkable cytotoxic activities. We will continue to explore other biological activities of multiflorane-type triterpenes.

\section{Supplementary Materials}

Supplementary materials can be accessed at: http://www.mdpi.com/1420-3049/19/4/4802/s1.

\section{Acknowledgments}

We thank Katsuhiko Minoura and Mihoyo Fujitake (Osaka University of Pharmaceutical Sciences) for NMR and MS measurements.

\section{Author Contributions}

T. Kikuchi performed the isolation, structure elucidation, and evaluation of bioactivities, and prepared the manuscript. S. Ueda, J. Kanazawa, and H. Naoe contributed to the isolation and structure elucidation. T. Yamada and R. Tanaka supervised whole research project.

\section{Conflicts of Interest}

The authors declare no conflict of interest.

\section{References}

1. Okada, M.; Mitsuhashi, H. Newly Revised Illustrated Medicinal Plants of World; Hokuryukan Pablishing Co., Ltd.: Tokyo, Japan, 2002; p. 514.

2. Chevallier, A. The Encyclopedia of Medicinal Plants; Seibundo Shinkosha Pablishing Co., Ltd.: Tokyo, Japan, 2000; p. 194.

3. Appendino, G.; Jakupovic, J.; Belloro, E.; Marchesini, A. Secondary metabolites from edible plants and spices. Part 4. Multiflorane triterpenoid esters from pumpkin. An unexpected extrafolic source of PABA. Phytochemistry 1999, 51, 1021-1026.

4. Appendino, G.; Jakupovic, J.; Belloro, E.; Marchesini, A. Triterpenoid p-aminobenzoates from the seeds of zucchini. Fitoterapia 2000, 71, 258-263.

5. Akihisa, T.; Ghosh, P.; Thakur, S.; Rosenstein, F.U.; Tamura, T.; Matsumoto, T. Widespread occurrence of cucurbita-5,24-dienol in Cucurbitaceae. Yukagaku 1986, 35, 1036-1040.

6. Cattel, L.; Balliano, G.; Caputo, O. Sterols and triterpenes from Cucurbita maxima. Planta Med. 1979, 37, 264-267.

7. Fenner, G.P.; Patterson, G.W.; Koines, P.M. Sterol composition during the life cycle of the soybean and the squash. Lipids 1986, 21, 48-51.

8. Fenner, G.P.; Patterson, G.W.; Lusby, W.R. Developmental regulation of sterol biosynthesis in Cucurbita. maxima L. Lipids 1989, 24, 271-277.

9. Kikuchi, T.; Takebayashi, M.; Shinto, M.; Yamada, T.; Tanaka, R. Three new multiflorane-type triterpenes from pumpkin (Cucurbita maxima) seeds. Molecules 2013, 18, 5568-5579. 
10. Ukiya, M.; Akihisa, T.; Tokuda, H.; Toriumi, M.; Mukainaka, T.; Banno, N.; Kimura, Y.; Hasegawa, J.; Nishino, H. Inhibitory effects of cucurbitane glycosides and other triterpenoids from the fruit of Momordica grosvenori on Epstein-Barr virus early antigen induced by tumor promoter 12-O-tetradecanoylphorbol-13-acetate. J. Agric. Food Chem. 2002, 50, 6710-6715.

11. De Marino, S.; Festa, C.; Zollo, F.; Iorizzi, M. Phenolic glycosides from Cucumis melo var. inodorus seeds. Phytochem. Lett. 2009, 2, 130-133.

12. De Shan, M.; Hu, L.H.; Chen, Z.L. A new multiflorane triterpenoid ester from Momordica cochinchinensis Spreng. Nat. Prod. Lett. 2001, 15, 139-145.

13. Ma, Y.P.; Li, N.; Gao, J.; Fu, K.L.; Qin, Y.; Li, G.Y.; Wang, J.H. A new peroxy-multiflorane triterpene ester from the processed seeds of Trichosanthes kirilowii. Helv. Chim. Acta 2011, 94, 1881-1887.

14. Akihisa, T.; Tokuda, H.; Ichiishi, E.; Mukainaka, T.; Toriumi, M.; Ukiya, M.; Yasukawa, K.; Nishino, H. Anti-tumor promoting effects of multiflorane-type triterpenoids and cytotoxic activity of karounidiol against human cancer cell lines. Cancer Lett. 2001, 173, 9-14.

15. Liu, C.H.; Yen, M.H.; Tsang, S.F.; Gan, K.H.; Hsu, H.Y.; Lin, C.N. Antioxidant triterpenoids from the stems of Momordica charantia. Food Chem. 2009, 118, 751-756.

16. Tanaka, R.; Kikuchi, T.; Nakasuji, S.; Ue, Y.; Shuto, D.; Igarashi, K.; Okada, R.; Yamada, T. A novel 3 $\alpha$-p-nitrobenzoylmultiflora-7:9(11)-diene-29-benzoate and two new triterpenoids from the seeds of zucchini (Cucurbita pepo L.). Molecules 2013, 18, 7448-7459.

17. Lin, J.W.; Chiang, H.M.; Lin, Y.C.; Wen, K.C. Natural products with skin-whitening effects. J. Food Drug Anal. 2008, 16, 1-10.

18. Lim, Y.J.; Lee, E.H.; Kang, T.H.; Ha, S.K.; Oh, M.S.; Kim, S.M.; Yoon, T.J.; Kang, C.; Park, J.H.; Kim, S.Y. Inhibitory effects of arbutin on melanin biosynthesis of $\alpha$-melanocyte stimulating hormone-induced hyperpigmentation in cultured Brownish guinea pig skin tissues. Arch. Pharm. Res. 2009, 32, 367-373.

19. Yamada, T.; Muroga, Y.; Jinno, M.; Kajimoto, T.; Usami, Y.; Numata, A.; Tanaka, R. New class azaphilone produced by a marine fish-derived Chaetomium globosum. The stereochemistry and biological activities. Bioorg. Med. Chem. 2011, 19, 4106-4113.

Sample Availability: Not available.

(C) 2014 by the authors; licensee MDPI, Basel, Switzerland. This article is an open access article distributed under the terms and conditions of the Creative Commons Attribution license (http://creativecommons.org/licenses/by/3.0/). 